\title{
La Maison brûle-t-elle? Une analyse d'une figure « enargique » dans les discours écologistes de Greta Thunberg
}

Sébastien Chonavey

\section{CpenEdition}

Journals

Édition électronique

URL : https://journals.openedition.org/rhetorique/1235

DOI : $10.4000 /$ rhetorique.1235

ISSN : 2270-6909

Éditeur

UGA Éditions/Université Grenoble Alpes

Édition imprimée

ISBN : 978-2-37747-325-0

Référence électronique

Sébastien Chonavey, « La Maison brûle-t-elle? Une analyse d'une figure « enargique » dans les discours écologistes de Greta Thunberg ", Exercices de rhétorique [En ligne], 17 | 2021, mis en ligne le 25 novembre 2021, consulté le 29 novembre 2021. URL : http://journals.openedition.org/rhetorique/ 1235 ; DOI : https://doi.org/10.4000/rhetorique.1235

Ce document a été généré automatiquement le 29 novembre 2021.

\section{c) ()ㅇㅇ (2)}

Les contenus de la revue Exercices de rhétorique sont mis à disposition selon les termes de la Licence Creative Commons Attribution - Pas d'Utilisation Commerciale - Partage dans les Mêmes Conditions 4.0 International. 


\title{
La Maison brûle-t-elle? Une analyse d'une figure « enargique » dans les discours écologistes de Greta Thunberg
}

\author{
Sébastien Chonavey
}

\section{Introduction : « notre maison brûle », brève histoire d'une image écologiste ${ }^{1}$}

Le 2 septembre 2002, devant l'assemblée plénière du quatrième Sommet de la Terre organisé par l'ONU, Jacques Chirac, réélu à la présidence de la République française prononce un discours qui obtient rapidement un retentissement mondial. En effet, souhaitant alerter l'opinion internationale sur l'urgence et la nécessité d'un combat politique qui prendrait enfin en compte les dégâts de la surexploitation de la nature sur l'humanité et son environnement, il avait prononcé les paroles suivantes :

Notre maison brûle et nous regardons ailleurs. La nature, mutilée, surexploitée, ne parvient plus à se reconstituer, et nous refusons de l'admettre. L'Humanité souffre. Elle souffre de mal développement, au Nord comme au Sud, et nous sommes indifférents. La Terre et l'humanité sont en péril, et nous sommes tous responsables (...) nous ne pourrons pas dire que nous ne savions pas. Prenons garde que le $\mathrm{XXI}^{\mathrm{e}}$ siècle ne devienne pas pour les générations futures celui d'un crime de l'humanité contre la vie.

Énumérant ainsi les grands problèmes environnementaux et de développement humain posés à l'ensemble des dirigeants et des opinions publiques mondiales, Jacques Chirac offrait une large diffusion à un constat effectué par de nombreux intellectuels, scientifiques et militants écologistes depuis plusieurs dizaines d'années. D'une façon remarquable, le discours avait frappé les esprits notamment grâce à la formule " Notre maison brûle et nous regardons ailleurs ». 
2 Probablement imaginée par Jean-Paul Deléage, un physicien spécialiste de géopolitique et conseiller de Jacques Chirac à cette époque, notamment sur la question environnementale, cette expression s'appuie sur un fonds culturel occidental antérieur que l'on peut retrouver dans plusieurs réalisations artistiques. Ces dernières peuvent ainsi relater l'incendie d'une maison dans la froideur, voire l'indifférence des propriétaires de celle-ci ainsi que d'autres spectateurs².

Dans la suite chronologique de ces précédentes utilisations composant un groupe de discours ou d'interventions culturelles marquées par le sceau de l'intertextualité, Greta Thunberg a effectué le 25 janvier 2019 une intervention remarquée devant l'assemblée du World Economic Forum de Davos, qui obtiendra un retentissement important tant dans la presse qu'auprès du grand public. Or ce discours reprenait à son compte une image décrivant la destruction de la planète et de son environnement sous la forme d'une maison en feu. Cette image est à nouveau exploitée par la jeune militante écologiste dans plusieurs allocutions ultérieures.

Souhaitant comprendre les raisons et les ressorts cette reprise, nous avons choisi d'analyser les usages de cette figure dans ses discours, et particulièrement l'exploitation qu'elle en propose dans son intervention au Parlement européen le 4 mars 2020, que nous proposons de rapprocher de l'antique ekphrasis. Afin de bien cerner les particularités de cette occurrence, nous commencerons notre enquête par un aperçu des autres mentions de la «maison qui brûle » dans les précédents discours de Greta Thunberg.

\section{La maison qui brûle dans les discours de Greta Thunberg}

5 La figure de la "maison qui brûle » est mobilisée dans sept discours prononcés ${ }^{3}$ par Greta Thunberg entre le 25 janvier 2019 et le 4 mars 2020, et il est intéressant de se demander si l'on peut observer une évolution dans son usage. C'est au World Economic Forum de Davos (discours 1) que la jeune activiste déclare pour la première fois que " notre maison est en feu » (" Our house is on fire »). Dans cette première attestation, la métaphore n'est que peu développée (il en est de même dans le discours qu'elle prononcera à New York; discours 6) : elle sert de point de départ pour engager un appel à l'action général face au changement climatique, que Greta Thunberg rend plus pressant encore en insistant sur la gravité de la situation, sur les conséquences désastreuses de la politique actuelle, mais en s'appuyant également sur les lieux du délibératif : la solution est simple ("even a small child can understand", "même un enfant peut comprendre »), vitale, et urgente. L'urgence et l'importance de la prise d'action sont soulignées dans la péroraison de son intervention, au sein de laquelle elle revient sur la métaphore de la maison qui brûle ${ }^{4}$ :

Adults keep saying: "we owe it to the young people to give them hope. » But I don't want you to hope. I don't want you to be hopeful. I want you to panic. I want you to feel the fear I feel every day. And then I want you to act. I want you to act as you would in a crisis. I want you to act as if the house was on fire. Because it is.

L'image sera reprise dans un second discours prononcé face à cette même assemblée en janvier 2020, soit un an plus tard, avec une intertextualité marquée ("One year ago I came to Davos and told you that our house is on fire »). 
6 La « maison qui brûle » est encore mobilisée en mars 2019 (discours 2), cette fois sur la scène de la cérémonie de remise de la Caméra d'Or à Berlin. Greta Thunberg s'adresse aux personnalités médiatiques, et les enjoint à faire comprendre aux dirigeants que « la maison est en feu » tout en insistant, par une anaphore, que "nous vivons dans un monde étrange » («We live in a strange world»), phrase qu'elle reprendra encore juste avant la péroraison finale. Si l'heure est grave, Greta Thunberg souligne, comme il est de coutume dans un discours délibératif, qu'il est encore temps d'agir ( We are failing but we have not yet failed. There is still time to fix this. It's up to us »).

7 En septembre 2019, la maison brûle encore. Greta Thunberg prend la parole à l'occasion de la grève pour le climat (discours 4), dans un contexte cette fois plus épidictique. Elle insiste en effet sur le fait que ce n'est pas seulement la maison des enfants qui est en feu ("Our house is on fire. And it's not just the young people's house. We all live here. It affects all for us and we won't just stand aside and watch. We are all united behind the science and we will do everything in our power to stop this crisis from getting worse ») et sur le fait que des adultes se sont joints au mouvement. Greta Thunberg propose ainsi un discours d'unité et de rassemblement ${ }^{5}$, entre les différents continents et les différentes générations.

8 On remarque un changement important dans son usage de la métaphore dans le discours qu'elle adresse, le 16 avril 2019, au Parlement européen de Strasbourg (discours 3). En effet, Greta Thunberg ouvre son intervention par une intertextualité avec le discours 1 : "My name is Greta Thunberg. I am 16 years old. I come from Sweden. And I want you to panic. I want you to act as if the house was on fire ". Cette intertextualité est parfaitement consciente et assumée ("I have said those words before, and a lot of people have explained why it is a bad idea ») et va servir désormais non pas à conscientiser la classe politique, mais à la blâmer pour son manque de réaction. Dans ce discours, la "maison qui brûle» est le point de départ de deux stratégies rhétoriques: premièrement, elle permet une comparaison aisée avec l'incendie de Notre-Dame, survenu la veille, en particulier dans l'idée que la cathédrale parisienne pourra être reconstruite (contrairement à notre planète) pour peu que ses fondations soient solides. Deuxièmement, elle lui permet de mettre en exergue l'absurdité de la situation. Après avoir décrit les conséquences $d u$ manque d'action face au dérèglement climatique en mobilisant différents rapports sur les conséquences chiffrées et illustrées à l'aide d'exemples concrets, elle s'engage dans une répétition anaphorique de la séquence "If our house was falling apart ", systématiquement suivie d'une réaction jugée non conforme à la situation et introduite par you wouldn't (par exemple : "If our world was falling apart, you wouldn't fly around the world in business class, chatting about how the market would solve everything with clever small solutions to specific isolated problems »). La potentielle puissance de l'image semble ici assumée et elle termine cette fois encore en rompant avec le mode hypothétique: "Well, our house is falling apart ", ce qui a pour effet de souligner l'ineptie du manque d'action politique, et, partant, de susciter l'indignation vis-à-vis des dirigeants. Une fois encore, son intervention se termine par un appel à l'action : répercuter la gravité de la situation et les voix des millions de jeunes qui ne peuvent pas voter dans les élections européennes à venir. Elle revient enfin sur deux idées amorcées en ouverture du discours : elle réexplore en particulier la comparaison établie avec Notre-Dame de Paris («It will take far-reaching vision, it will take courage, it will take fierce determination to act now, to lay the foundations where we may not know over details about how to shape the ceiling. In other words: it would take cathedralthinking »), en concédant le statut particulier de son expertise et en reprenant les 
indications personnelles données en introduction («It's okay if you refuse to listen to me. I am after all just a 16-year-old schoolgirl from Sweden »); elle redonne ainsi à ses interlocuteurs la position de force (politique) qu'ils exercent effectivement, tout en les plaçant face à leurs responsabilités, et accorde une importance plus grande encore aux données précises et chiffrées énoncées dans le cœur de son argumentation, ainsi qu’à l'évidente urgence accentuée par la métaphore de la maison qui s'écroule.

9 À Madrid, en décembre 2019 (discours 5), Greta Thunberg annonce renoncer aux images et phrases-chocs utilisées dans ces précédents discours. Elle dit :

A year and a half ago, I didn't speak to anyone unless I really had to. But then I found a reason to speak. Since then, I have given many speeches and learnt that when you talk in public, you should start with something personal or emotional to get everyone's attention. Say things like "Our house is on fire", "I want you to panic" or "How dare you ». But today I will not do that. Because then, those phrases are all that people focus on. They don't remember the facts, the very reasons why I say those things in the first place. We no longer have time to leave out the science.

Ce revirement a priori drastique, souligné par un usage important d'expressions métadiscursives, s'accompagne d'un changement d'ethos, puisque la jeune femme assume ici une position d'experte et une posture professorale. Cela passe essentiellement, du moins dans la première moitié du discours, par une énonciation de chiffres dûment, voire froidement, référencés (ex.: "In chapter 2, on page 108, in the SR 1.5 IPCC report [...] »). L'écart par rapport aux discours précédents est tel, même dans son énonciation des données chiffrées, que l'on en viendrait presque à se demander s'il ne faut pas voir ici une forme de discours figuré ${ }^{6}$ : tout en déclarant renoncer aux métaphores pour se concentrer sur les faits, Greta Thunberg insiste sur la nécessité de ressentir la peur pour passer à l'action. Plusieurs éléments peuvent être avancés pour soutenir une telle hypothèse, à commencer par la prétérition initiale (puisqu'elle ouvre, précisément, son discours par une information personnelle). Insistons aussi sur la manière particulièrement formelle et factuelle dont elle introduit les données chiffrées, qui se démarque de ses précédents discours, et, surtout, sur l'usage qu'elle fait malgré tout de la métaphore dans son discours : si elle renonce ici à la maison qui brûle, elle met en parallèle une réaction attendue face à un enfant en danger de mort au milieu d'une route et ce que les dirigeants devraient faire face à l'urgence climatique, soulignant, une nouvelle fois, l'aberration de leur attitude ${ }^{7}$.

10 Si notre but n'est pas ici de caractériser une fois pour toutes la rhétorique déployée par Greta Thunberg, il convient à ce stade de tirer plusieurs observations générales des discours susmentionnés :

- Au niveau formel, nous soulignerons un soin presque scolaire apporté à la composition des discours et un goût marqué pour les structures circulaires et les anaphores.

- Elle assume explicitement le pathos de ses discours, car il est nécessaire, selon elle, de ressentir la peur, de paniquer, pour passer à l'action; émotions auxquelles l'image de la maison qui brûle semble particulièrement associée.

- On constate une évolution de l'ethos de l'oratrice, qui passe du statut d'enfant porte-parole (en particulier à Strasbourg) à celui d'experte ${ }^{8}$.

- Enfin, Greta Thunberg semble favoriser l'usage de comparaisons et de métaphores (maison en feu, maison sur le point de s'écrouler; comparaison avec Notre-Dame ou avec un enfant en danger sur la route) pour rendre évidents l'inconscience des dirigeants politiques et le péril de la situation. 


\section{La maison qui brûle, une métaphore « énargique » ?}

11 Au moment où Greta Thunberg s'avance, le 4 mars 2020, face à la tribune du Parlement européen dans le cadre de la commission environnement (discours 7), l'image de la maison qui brûle est déjà entrée dans son arsenal rhétorique. L'usage qu'elle en fait dans ce discours se démarque toutefois des discours précédents par la description qu'elle va y opérer; alors que la métaphore n'était pas ou peu développée dans les attestations précédentes, Greta Thunberg va ici lui donner vie :

You said that the EU would lead against the existential threat of the climate crisis. And this was wonderful news. When your children set off the fire alarm, you went outside, took a look and smell the air. You stated that, yes, the house is actually burning. This was no false alarm. But then you went back inside, finished your dinner and watched your movie and went to bed without even calling the fire department. I'm sorry, but this makes no sense at all when your house is on fire. You don't wait a few more years to start putting it out.

Si ce développement est en quelque sorte préparé par les occurrences et discours précédents, il convient de s'intéresser au fonctionnement propre de cet usage particulier et à ses effets. Nous proposons ici de rapprocher cette utilisation de l'ancienne technique de l'ekphrasis. Les traités de rhétorique définissent l'ekphrasis comme une description "vivante", qui place sous les yeux des lecteurs ou des auditeurs l'objet décrit ${ }^{9}$. Il est aisé de se représenter la première scène: le son de l'alarme incendie, les adultes qui sortent pour constater que la maison est en flammes, l'odeur de brûlé. Cette situation familière appelle une réaction évidente, car comprise dans une sorte de «script »: tenter d'éteindre l'incendie. Or, c'est précisément ce que ne font pas les adultes. La seconde partie de la description vient souligner cette rupture par rapport aux attentes sur le mode de l'absurde : les adultes retournent à l'intérieur de la maison, terminent leur repas, regardent un film et vont se coucher sans se préoccuper du feu. Ceci rappelle bien sûr l'anaphore "If our house was falling apart, you wouldn't... ", mobilisée lors du discours de Strasbourg, ou encore la comparaison entre l'urgence climatique à laquelle nous devons faire face et le danger encouru par un enfant qui se trouverait au milieu de la route alors que des voitures arrivent à toute allure. Ici encore, face à un auditoire de politiciens, Greta Thunberg fustige par l'absurde leur manque de réaction. Mais cette fois, elle cherche, pour ainsi dire, à mettre cette absurdité sous les yeux de ses auditeurs. Ils se représentent aisément la scène, imaginent peut-être même les flammes visibles à travers les vitres de la maison, ainsi que le danger qui guette ses habitants. De ce point de vue, cet extrait de discours s'apparente au procédé rhétorique de l'ekphrasis, telle qu'il est décrit dans les manuels et traités remontant à l'Antiquité. La description de cette scène est suffisamment topique ou familière pour que chacun puisse se la représenter et suscite à la fois la peur pour les habitants de la maison, l'incompréhension face au manque de réaction des adultes, mais aussi la colère envers ceux qui n'agissent pas, et mettent ainsi leur vie et celle des enfants en danger, alors qu'ils ont les moyens d'intervenir.

Il y a cependant deux obstacles à voir une ekphrasis à proprement parler dans cette figure. Premièrement, il s'agit d'une métaphore : elle ne décrit donc pas l'objet dont il est question lui-même. Deuxièmement, alors que la théorie rhétorique insiste sur l'importance de la vraisemblance, la scène décrite par Greta Thunberg est absurde. Or, une ekphrasis n'est, en théorie, pas censée représenter quelque chose qui n'existe pas. Dans la suite de cette contribution, nous nous proposons donc de questionner la pertinence de lui adosser l'étiquette "ekphrasis», ainsi que les effets et limites de cet 
usage. Les difficultés que nous venons de soulever ne sont toutefois pas insurmontables. En effet, les comparaisons ne sont pas absentes des ekphraseis antiques, selon les principes bien connus, qu'un objet familier et concret est plus facile à se représenter ${ }^{10}$, et que les ekphraseis elles-mêmes doivent prendre appui, pour être efficaces, sur des images topiques ${ }^{11}$. L'on peut également s'inspirer d'une réflexion d'Aristote, au troisième livre de la Rhétorique :

Mais il faut dire aussi ce que nous entendons par « mise sous les yeux » et comment on fait pour que cela se produise. Je dis que met sous les yeux tout ce qui désigne un être en action. [...] Il y a aussi cette façon de s'exprimer dont use souvent Homère, qui consiste à rendre animés les inanimés grâce à la métaphore ${ }^{12}[\ldots]$.

Or il faut bien garder à l'esprit que Greta Thunberg ne cherche pas seulement à mettre en garde contre les dangers du changement climatique, qui pourraient faire l'objet d'une description plus concrète (forêt en feu, inondations, etc.), mais aussi à souligner l'absurdité du manque d'action politique, qui ne peut que difficilement s'exprimer en termes d'images concrètes ou de chiffres ${ }^{13}$.

Pour ce qui concerne le deuxième écueil, et l'absurdité de la scène, soulignons que si le ridicule et l'absurde sont généralement absents de l'usage "progymnastique " de l'ekphrasis, ils sont toutefois attestés chez des rhéteurs chevronnés de l'Antiquité, notamment dans les déclamations : pensons en particulier à la déclamation $\mathrm{du}$ " mur marqué de traces de mains ", dans laquelle un fils aveugle est accusé par sa belle-mère d'avoir assassiné son père pendant la nuit, et où le déclamateur tente d'imaginer de façon "enargique " comment l'aveugle aurait pu commettre le meurtre afin de montrer l'absurdité de la position adverse ${ }^{14}$.

Enfin, et c'est sans doute là le point le plus important: on ne peut nier l'effet «enargique » de cette métaphore. Par le développement de celle-ci, Greta Thunberg allie les effets d'évidence de la comparaison et de l'ekphrasis, qui s'invitent ainsi comme adjuvants des données chiffrées. Mettre sous les yeux des auditeurs une maison en feu suscite la crainte face un danger bien présent, certainement plus aisé à se représenter que les effets futurs du dérèglement climatique, ce qui est cohérent avec la conviction exprimée par Greta Thunberg qu'il faut que la communauté internationale panique pour agir. Mais cette description " enargique » suscite aussi, et à notre sens surtout, l'indignation face à des dirigeants qui n'agissent pas. Il est d'ailleurs significatif que les enfants ne sont plus mentionnés après qu'ils ont donné l'alerte.

L'effet " enargique " de la métaphore nous semble d'autant plus frappant que cette partie du discours du 4 mars 2020 a fait l'objet d'une mise en scène cinématographique brève. En effet, le 22 avril 2010, "Fridays For Future ", le mouvement de grève scolaire pour le climat dirigé par des « jeunes » proches de Greta Thunberg, a publié une vidéo qui illustrait la vie d'une famille vivant dans une maison en feu. On y voit le réveil et les préparatifs du matin (petit-déjeuner, soins des dents et habillement) s'effectuer apparemment de manière paisible et heureuse alors que, dans le même temps, l'extérieur de la maison ainsi que les pièces concernées sont en proie aux flammes.

17 La mise sous le format vidéo permet ici aux créateurs d'amplifier encore le procédé " énargique » en plaçant littéralement sous les yeux de l'auditoire le caractère absurde de la situation développée dans le discours de Greta. Si, bien entendu, c'est l'aspect visuel de la situation qui est mis en avant dans le court-métrage, la vidéo vient, comme dans les exemples traditionnels de l'ekphrasis prototypique, renforcer des éléments plurisensoriels voire synesthésiques déjà présents dans le discours originel («took a look 
and smell the air " et "then you went back inside, finished your dinner and watched your movie »). Sur ce point, plusieurs éléments sont mis en avant. Il en va ainsi du son du crépitement et de l'intensité des flammes délibérément augmentés par rapport aux brefs dialogues présents, des sensations éprouvées par le spectateur aux souvenirs du contact avec le feu (sensations développées à l'écran par le contact des personnages avec des objets enflammés), du goût de la nourriture associée aux traces de cendres et surtout de la fumée qui occupe le fond et une bonne partie de l'image. Le clip se termine sur un appel à l'action réalisé sobrement sur fond d'une image blanche et du son du crépitement des flammes : "Our house is on fire. React ${ }^{15}$ » et qui vient là encore appuyer les demandes déjà présentes dans les discours de Greta Thunberg au sujet de décisions débouchant sur des actions concrètes.

18 Il s'agit de la déclinaison la plus aboutie de la "métaphore énargique " effectuée à partir de "our house is on fire ", c'est aussi la dernière fois que Greta Thunberg aura recours - jusqu'à aujourd'hui - à l'emploi de ce procédé.

\section{Conclusions : une stratégie de l'évidence ou « Un je t'aime moi non plus » à la rhétorique?}

19 En conclusion, la question rhétorique qui ressort de notre analyse est celle de la stratégie adoptée par la jeune militante écologiste.

Peut-on tout d'abord parler de stratégie à proprement parler? Il semble incontestablement que oui, et notamment lorsqu'on relie cette question aux objectifs de prise de conscience et de nécessité de passage à l'action énoncés de manière constante par Greta Thunberg elle-même dans ses premières prises de parole ${ }^{16}$. La stratégie adoptée rencontre-t-elle pour autant les effets escomptés ? C'est une question plus délicate à laquelle il convient de répondre de façon ouverte pour poursuivre un chantier d'analyse qui s'avère plus complexe qu'on aurait pu le croire. En effet, visiblement, Greta Thunberg cherche à construire un effet d'enargeia, consciente que l'effet d'évidence qui place une réalité absente sous les yeux de l'auditoire peut avoir un grand pouvoir mobilisateur. Mais on peut percevoir comme une hésitation entre deux stratégies rhétoriques. Le discours opte tantôt pour la stratégie de l'évidence "discursive ", en choisissant la métaphore ekphrastique de la maison qui brûle, tantôt pour la preuve extra-technique des chiffres et statistiques, en somme, le recours à l'évidence au sens anglais du terme evidence. Plutôt que de qualifier cette stratégie de " maladroite ${ }^{17}$ ", il est sans doute plus prudent d'y voir une forme d'ambivalence qui, chez la jeune oratrice, peut, comme nous l'avons vu, s'incarner de manière paradoxale dans la mise en place d'ekphrasis censément développée par des éléments de descriptions extra-techniques.

21 Cette ambivalence paradoxale semble d'autant plus importante chez Greta Thunberg que celle-ci pourrait être renforcée par un ethos pris lui aussi entre la figure de l'enfant et celle de l'expert. Si, de ce point de vue, il sera crucial de saisir l'évolution de ses stratégies rhétoriques dans l'avenir, il faudra pourvoir également s'attarder sur la multiplication dans ses discours d'appropriations tâtonnantes et non abouties de stratégies discursives venues d'autres orateurs reconnus ${ }^{18}$. En effet, ce mouvement, analysé comme un phénomène transversal à toute la discipline, pourrait témoigner, dans le chef de l'orateur et de son auditoire, d'une incompétence rhétorique plus large 
- incompétence que pourrait venir combler le choix de la réalisation d'une vidéo, recoupant l'importance prise par l'image dans la culture contemporaine.

En définitive, le recours à l'image de la maison qui brûle nous semble encore relever d'une stratégie classiquement délibérative qui recommande un passage à l'action. Mais on voit aussi apparaitre des éléments qui relèvent davantage d'un blâme collectif. Celui-ci pourrait-il déboucher sur une nouvelle forme d'épidictique aux accents nihilistes? L'avenir nous le dira.

\section{ANNEXES}

\section{Discours de Greta Thunberg (extraits avec traduction)}

Le lecteur trouvera ci-dessous les extraits de discours de Greta Thunberg faisant intervenir la métaphore de la maison qui brûle, et qui ont servi de support au présent article. Les retranscriptions, traductions et soulignements sont personnels.

\section{25 janvier 2019 : Forum de Davos (I)}

Our house is on fire. I'm here to say our house is on fire.

According to the IPCC we are less than twelve years away from not being able to undo our mistakes. At that time, unprecedented changes in all aspects of society need to have taken place. Including a reduction of power $\mathrm{CO} 2$ emissions by at least fifty percent. And please note that those numbers do not include the aspects of equity which is absolutely necessary to make the Paris agreement work on a global scale. Nor does it include tipping points or feedback loops like the extreme powerful methane gas being released from the thawing arctic permafrosts. [...]

Notre maison est en feu. Je suis ici pour dire que notre maison est en feu.

Selon le GIEC, nous sommes à moins de douze ans de ne plus pouvoir réparer nos erreurs. À ce moment-là, des changements sans précédent devront avoir eu lieu dans tous les aspects de la société. Y compris une réduction des émissions de $\mathrm{CO} 2$ de l'énergie d'au moins cinquante pour cent. Et notez bien que ces chiffres n'incluent pas la question de l'équité qui est absolument nécessaire pour que l'accord de Paris fonctionne à l'échelle mondiale. Ils n'incluent pas non plus les points de basculement ou les boucles de rétroaction comme le méthane extrêmement puissant libéré par le dégel du permafrost arctique. [...]

Adults keep saying we owe to the young people to give them hope. But I don't want your hope. I don't want to be hopeful. I want you to panic. I want you to feel the fear that I feel every day. And then I want to act. I want to act as we would be in a crisis. I want to act as if the house was on fire because it is. Thank you.

Les adultes n'arrêtent pas de dire que nous devons donner de l'espoir aux jeunes. Mais je ne veux pas de votre espoir. Je ne veux pas être pleine d'espoir. Je veux que vous paniquiez. Je 
veux que vous ressentiez la peur que je ressens chaque jour. Et ensuite je veux agir. Je veux agir comme nous le ferions en cas de crise. Je veux agir comme si la maison était en feu parce qu'elle l'est. Merci.

\section{30 mars 2019 - Remise de la Caméra d'Or à Berlin}

People see you celebrities as gods. You influence billions of people. We need you. You can use your voice to raise awareness about this global crisis. You can help turn individuals into movements. You can help us wake up our leaders - and let them know that our house is on fire.

We live in a strange world. But it's the world that my generation has been handed. It's the only world we've got. We are now standing at a crossroads in history. We are failing but we have not yet failed. We can still fix this. It's up to us.

Les gens vous voient, vous les célébrités, comme des dieux. Vous influencez des milliards de personnes. Nous avons besoin de vous. Vous pouvez utiliser votre voix pour sensibiliser les gens à cette crise mondiale. Vous pouvez aider à transformer des individus en mouvements. Vous pouvez nous aider à réveiller nos dirigeants - et leur faire savoir que notre maison est enfeu.

Nous vivons dans un monde étrange. Mais c'est le monde que ma génération a reçu. C'est le seul monde que nous ayons. Nous nous trouvons maintenant à un carrefour de l'histoire. Nous sommes en train d'échouer, mais nous n'avons pas encore échoué. Nous pouvons encore réparer ça. Il ne tient qu'à nous de le faire.

\section{16 avril 2019 - Discours au parlement européen de Strasbourg}

My name is Greta Thunberg. I am 16 years old. I come from Sweden and I want you to panic. I want you to act as if your house was on fire. I have said those words before. And a lot of people have explained why that is a bad idea. A great number of politicians have told me that panic never leads to anything good. And I agree. To panic unless you have to is a terrible idea.

But when your house is on fire and you want to keep your house from burning to the ground, then that does require some level of panic. Our civilisation is so fragile. It is almost like a castle built in the sand. The facade is so beautiful. But the foundations are far from solid. We have been cutting so many corners. Yesterday the world watched with despair and enormous sorrow how the Notre Dame burnt in Paris. Some buildings are much more than just buildings. But the Notre Dame will be rebuilt. I hope that its foundations are strong. I hope that our foundations are even stronger. But I fear that they are not. [...]

Je m'appelle Greta Thunberg. Je suis âgée de 16 ans. Je viens de Suède et je veux que vous paniquiez. Je veux que vous agissiez comme si votre maison était en feu. J'ai déjà dit ces mots auparavant. Et beaucoup de gens m'ont expliqué pourquoi c'était une mauvaise idée. Un grand nombre de politiciens m'ont dit que la panique ne mène jamais à rien de bon. Et je suis d'accord. Paniquer à moins d'y être obligé est une très mauvaise idée.

Mais lorsque votre maison est en feu et que vous voulez éviter qu'elle ne soit réduite en cendres, il faut un certain degré de panique. Notre civilisation est si fragile. C'est presque comme un château construit dans le sable. La façade est si belle. Mais les fondations sont loin d'être solides. Nous avons pris tellement de libertés. Hier, le monde a regardé avec désespoir et une énorme tristesse comment Notre-Dame a brûlé à Paris. Certains bâtiments sont bien plus que de simples bâtiments. Mais Notre-Dame sera reconstruite. J'espère que ses fondations sont solides. J'espère que nos fondations sont encore plus solides. Mais je crains qu'elles ne le soient pas. [...] 
And our leaders need to start acting accordingly. Because at the moment they are not. If our house was falling apart, our leaders wouldn't go on like you do today. You would change almost every part of your behaviour. As you do in an emergency.

If our house was falling apart, you wouldn't fly around the world in business class chatting about how the market will solve everything with clever small solutions to specific isolated problems. You wouldn't talk about buying and building your way out of a crisis that has been created by buying and building things.

If our house was falling apart, you wouldn't hold 3 emergency Brexit summits and no emergency summit regarding the breakdown of the climate and ecosystems. You wouldn't be arguing about phasing out coal in 15 or 11 years.

If our house was falling apart, you wouldn't be celebrating that one single nation like Ireland may soon divest from fossil fuels. You wouldn't celebrate that Norway has decided to stop drilling for oil outside the scenic resort of Lofoten Island, but will continue to drill everywhere else for decades. It's 30 years too late for that kind of celebrations

If our house was falling apart, the media wouldn't be writing about anything else. The ongoing climate- and ecological crises would make up all the headlines.

If our house was falling apart, you wouldn't say that you have the situation under control and place the future living conditions for all living species in the hands of inventions that are yet to be invented. And you would not spend all your time as politicians arguing over taxes or Brexit.

If the walls of our house truly came tumbling down, surely you would set your differences aside and start cooperating.

Well, our house is falling apart. And we are rapidly running out of time. [...]

Et nos dirigeants doivent commencer à agir en conséquence. Car pour l'instant, ils ne le font pas. Si notre maison s'écroulait, nos dirigeants ne continueraient pas comme ils le font aujourd'hui. Vous changeriez presque tous les aspects de votre comportement. Comme vous le faites en cas d'urgence.

Si notre maison s'écroulait, vous ne feriez pas le tour du monde en classe affaires en discutant de la façon dont le marché va tout résoudre grâce à de petites solutions intelligentes à des problèmes spécifiques isolés. Vous ne parleriez pas d'acheter et de construire pour sortir d'une crise qui a été créée en achetant et en construisant.

Si notre maison était en train de s'effondrer, vous ne tiendriez pas trois sommets d'urgence sur le Brexit et aucun sommet d'urgence sur l'effondrement du climat et des écosystèmes. Vous ne seriez pas en train de discuter de l'élimination progressive du charbon dans 15 ou 11 ans.

Si notre maison était en train de s'effondrer, vous ne vous réjouiriez pas qu'une seule nation comme l'Irlande puisse bientôt renoncer aux combustibles fossiles. Vous ne vous réjouiriez pas que la Norvège ait décidé d'arrêter les forages pétroliers en dehors de la station balnéaire de l'île de Lofoten, mais qu'elle continue à forer partout ailleurs pendant des décennies. Ce genre de célébrations ont 30 ans de retard.

Si notre maison était en train de s'effondrer, les médias ne parleraient pas d'autre chose. Les crises climatiques et écologiques en cours feraient tous les gros titres.

Si notre maison s'écroulait, vous ne diriez pas que vous maîtrisez la situation et que vous placez les conditions de vie future de toutes les espèces vivantes entre les mains d'inventions qui restent à inventer. Et vous ne passeriez pas tout votre temps en tant que politiciens à vous disputer sur les impôts ou le Brexit. 
Si les murs de notre maison s'écroulaient vraiment, vous mettriez sûrement vos différences de côté et commenceriez à coopérer.

Eh bien, notre maison est en train de s'écrouler. Et le temps nous manque cruellement. [...]

Our house is falling part. The future - as well as all that we have achieved in the past is literally in your hands now. But it is not too late to act. It will take a far-reaching vision. It will take courage. It will take a fierce determination to act now to lay the foundations when we may not know all of the details about how to shape the ceiling. In other words, it will take « cathedral thinking».

I ask you to please wake up and make the required changes possible. To do your best is no longer good enough. We must all do the seemingly impossible. And it's okay if you refuse to listen to me. I am after all just a 16-year-old schoolgirl from Sweden. But you cannot ignore the scientists. Or the science. Or the millions of schoolchildren school striking for the right to a future. I beg you : please do not fail on this.

Notre maison est en train de s'écrouler. L'avenir - ainsi que tout ce que nous avons réalisé dans le passé - est littéralement entre vos mains maintenant. Mais il n'est pas trop tard pour agir. Il faudra une vision ambitieuse. Il faudra du courage. Il faudra une détermination farouche pour agir maintenant afin de poser les fondations alors que nous ne connaissons peut-être pas tous les détails sur la manière de façonner le plafond. En d'autres termes, il faudra adopter une "pensée cathédrale ".

Je vous demande de vous réveiller, s'il vous plait, et de rendre possibles les changements nécessaires. Faire de son mieux n'est plus suffisant. Nous devons tous faire ce qui semble impossible. Et ce n'est pas grave si vous refusez de m'écouter. Après tout, je ne suis qu'une écolière suédoise de 16 ans. Mais vous ne pouvez pas ignorer les scientifiques. Ou la science. Ou les millions d'écoliers en grève pour le droit à un avenir. Je vous en prie : s'il vous plaît, n'échouez pas sur ce point.

\section{20 septembre 2019 - Grève mondiale du climat à New York}

And we are not in school today. This time we are not alone. We have some adults who are not at work today either. And why ? Because this is an emergency, our house is on fire. And it's not just the young people's house, we all live here. It affects all of us and we will not just stand aside and watch. We are united behind science and we will do everything in our power to stop this crisis from getting worse.

Et nous ne sommes pas à l'école aujourd'hui. Cette fois nous ne sommes pas seuls. Nous avons des adultes qui ne sont pas au travail aujourd'hui non plus. Et pourquoi? Parce que c'est une urgence, notre maison est en feu. Et ce n'est pas seulement la maison des jeunes, nous vivons tous ici. Nous sommes tous concernés et nous n'allons pas nous contenter de rester à l'écart et de regarder. Nous sommes unis derrière la science et nous ferons tout ce qui est en notre pouvoir pour empêcher cette crise de s'aggraver.

\section{11 décembre 2019 - Conférence des Nations Unies sur le climat à Madrid}

Hi. A year and a half ago, I didn't speak to anyone unless I really had to, but then I found a reason to speak. Since then, I've given many speeches and learned that when you talk in public, you start with something personal or emotional to get everyone's attention. Say things like, « our house is on fire », «I want you to panic » or « How dare you ».

But today I will not do that because then those phrases are all that people focus on. They don't remember the facts, the very reason why I said those things in the first place. We no longer have time to leave out the science. For about a year I have been 
constantly talking about our rapidly declining carbon budgets over and over again. But since that is still being ignored, I will just keep repeating it.

Bonjour. Il y a un an et demi, je ne parlais à personne, sauf si je devais vraiment le faire, mais j'ai ensuite trouvé une raison de parler. Depuis, j'ai fait de nombreux discours et j'ai appris que lorsque vous parlez en public, vous devez commencer par quelque chose de personnel ou d'émotionnel pour attirer l'attention de tous. Dire des choses comme " maison est en feu ", "je veux que vous paniquiez » ou " comment osez-vous".

Mais aujourd'hui, je ne le ferai pas, car les gens ne retiennent que ces phrases. Ils ne se souviennent pas des faits, de la raison même pour laquelle j'ai dit ces choses en premier lieu. Nous n'avons plus le temps de laisser de côté la science. Car depuis environ un an, je ne cesse de parler de la diminution rapide de nos budgets carbone, encore et encore. Mais puisque cela est toujours ignoré, je vais continuer à le répéter.

\section{21 janvier 2020 - Forum de Davos (II)}

One year ago I came to Davos and told you that our house is on fire. I said I wanted you to panic. I've been warned that telling people to panic about the climate crisis is a very dangerous thing to do. But don't worry. It's fine. Trust me, I've done this before and I can assure you it doesn't lead to anything. And, for the record, when we children tell you to panic we're not telling you to go on like before. [...]

Il y a un an, je suis venue à Davos et je vous ai dit que notre maison était en feu. J'ai dit que je voulais que vous paniquiez. On m'a prévenue que dire aux gens de paniquer à propos de la crise climatique est une chose très dangereuse à faire. Mais ne vous inquiétez pas. C'est bon. Faites-moi confiance, je l'ai déjà fait et je peux vous assurer que cela ne mène à rien. Et, pour mémoire, quand nous, les enfants, vous disons de paniquer, nous ne vous disons pas de continuer comme avant. [...]

From a sustainability perspective, the right, the left as well as the centre have all failed. No political ideology or economic structure has been able to tackle the climate and environmental emergency and create a cohesive and sustainable world. Because that world, in case you haven't noticed, is currently on fire.

You say children shouldn't worry. You say : « Just leave this to us. We will fix this, we promise we won't let you down. Don't be so pessimistic.» And then, nothing. Silence. Or something worse than silence. Empty words and promises which give the impression that sufficient action is being taken. [...]

Du point de vue de la durabilité, la droite, la gauche et le centre ont tous échoué. Aucune idéologie politique ou structure économique n'a été capable de s'attaquer à l'urgence climatique et environnementale et de créer un monde cohérent et durable. Parce que ce monde, au cas où vous ne l'auriez pas remarqué, est actuellement en feu.

Vous dites que les enfants ne devraient pas s'inquiéter. Vous dites: "Laissez-nous faire. Nous allons arranger ça, nous promettons de ne pas vous laisser tomber. Ne soyez pas si pessimiste». Et puis, rien. Le silence. Ou quelque chose de pire que le silence. Des paroles vides et des promesses qui donnent l'impression que des mesures suffisantes sont en train d'être prises. [...]

Our house is still on fire. Your inaction is fuelling the flames by the hour. And we are telling you to act as if you loved your children above all else. Thank you.

Notre maison est toujours en feu. Votre inaction alimente les flammes d'heure en heure. Et nous vous disons d'agir comme si vous aimiez vos enfants par-dessus tout. Merci. 


\section{4 mars 2020 - Parlement européen, dans le cadre de la Commission Environnement}

You said that the EU would lead against the existential threat of the climate crisis. And this was wonderful news. When your children set off the fire alarm, you went outside, took a look and smell the air. You stated that, yes, the house is actually burning.

This was no false alarm. But then you went back inside, finished your dinner and watched your movie and went to bed without even calling the fire department. I'm sorry, but this makes no sense at all when your house is on fire. You don't wait a few more years to start putting it out.

And yet this is what the commission is proposing today. When the EU presents this climate law and net zero by 2050 , you indirectly admit surrender : that you are giving up, giving up on the Paris agreement, giving up on your promises and giving up on doing everything you possibly can to ensure a safe future for your own children. [...]

Vous avez dit que l'UE prendrait la tête du combat contre la menace existentielle que représente la crise climatique. Et c'était une merveilleuse nouvelle. Lorsque vos enfants ont déclenché l'alarme incendie, vous êtes sorti, vous avez regardé et senti l'air. Vous avez déclaré que, oui, la maison brûle réellement.

Ce n'était pas une fausse alerte. Mais ensuite vous êtes retournés à l'intérieur, avez fini votre dîner, regardé votre film et êtes allés vous coucher sans même appeler les pompiers. Je suis désolé, mais cela n'a aucun sens quand votre maison est en feu. Vous n'attendez pas quelques années de plus pour commencer à l'éteindre.

Et pourtant, c'est ce que la Commission propose aujourd'hui. Lorsque l'UE présente cette loi sur le climat et le "net zéro" d'ici 2050, vous admettez indirectement que vous capitulez: que vous renoncez, que vous renoncez à l'accord de Paris, que vous renoncez à vos promesses et que vous renoncez à faire tout ce qui est en votre pouvoir pour assurer un avenir sûr à vos propres enfants. [...]

Anything else is surrender. This climate law is surrender because nature doesn't bargain and you cannot make deals with physics. And we will not allow you to surrender on our future.

Thank you.

Tout le reste est une capitulation. Cette loi sur le climat est une capitulation parce qu'on ne négocie pas avec la nature et que vous ne pouvez pas conclure de marché avec la physique. Et nous ne vous permettrons pas de capituler sur notre avenir. Merci.

\section{NOTES}

1. L'auteur tient à remercier chaleureusement le Prof. Emmanuelle Danblon, Julie Dainville, Lucie Donckier de Donceel et Benoît Sans sans lesquels ni l'écriture du présent article ni sa parution n'auraient pu voir le jour.

2. Sans en faire une généalogie précise, c'est par exemple le cas dans le domaine littéraire (Histoire de la maison qui brûle de France Daigle en 1985 ou Vendredi ou la vie sauvage de Michel Tournier en 1971), mais également en musique (Beds are Burning de Midnight Oil en 1986 ou Dans le Nord Canadien de Zachary Richard en 1996).

3. Les extraits sont fournis en annexe.

4. Nous donnons en annexe la traduction française des extraits cités. - Notons ici que, dans cette seconde occurrence au sein du même discours, la jeune oratrice reprend l'image sur le mode du « comme si » (as if), se montrant ainsi consciente de son caractère poétique. 
5. Sur cet aspect du genre épidictique, voir notamment Ch. PERELMAN et L. OLBRECHTS-TYTECA, Traité de l'argumentation. La nouvelle rhétorique, Bruxelles, Éditions de l'Université de Bruxelles, 1958, p. 62-68 ; E. DANBLON, «La rationalité du discours épidictique », dans M. DOMINICY et M. FRÉDÉRIC (éds.), La mise en scène des valeurs. La rhétorique de l'éloge et du blâme, Lausanne - Paris, Delachaux et Niestlé, 2001, p. 19-47.

6. P. CHIRON, «Le logos eskhèmatisménos ou discours figuré ", dans G. DECLERCQ, M. MURAT et J. DANGEL (éd.), La parole polémique, Paris, Champion, 2003, p. 223-254.

7. "If there is a child standing in the middle of the road and cars are coming at full speed, you don't look away because it's too uncomfortable. You immediately run out and rescue that child. And without that sense of urgency, how can we, the people understand that we are facing a real crisis ".

8. Pour cette évolution de l'éthos de l'enfant vers d'autres formes, voir S. CHONAVEY, « Les figures de l'écologie: vers une nouvelle incarnation de la jeunesse face aux adultes. Une analyse de l'ethos dans les discours écologistes à l'ONU de Greta Thunberg, Felix Finkbeiner et Severn CullisSuzuki entre 1992 et 2019 ", communication au colloque du GER CESS "Comment parler d'environnement? » organisé le 10 décembre 2020 à Aix-en-Provence, à paraître.

9. Il s'agit plus précisément de la définition proposée par les manuels de progymnasmata; pour une synthèse et un commentaire sur l'ekphrasis en tant que progymnasma, voir notamment P. CHIRoN, Manuel de rhétorique ou Comment faire de l'élève un citoyen, Paris, Les Belles Lettres, 2019 ; et l'introduction de ce volume.

10. Voir par exemple Quintilien VIII, 3, 72-73. Voir notamment R. WEBB, Ekphrasis, Imagination and Persuasion in Ancient Rhetorical Theory and Practice, Farnham, Ashgate, 2009 [2012], en particulier p. 110-113.

11. Voir à ce propos les exemples cités dans l'article de B. Sans, dans le présent volume.

12. Aristote, Rhétorique III, 11, 1411b24-35 ; trad. : P. CHIRON, 2007.

13. Danielle van Mal-Maeder relève, dans le même ordre d'idée, des descriptions métaphoriques du processus de création littéraire dans les déclamations (La fiction des déclamations, Leyde Boston, Brill, 2007, p. 85-86).

14. Ps.-Quintilien, Decl. Mai., 1, 9 et le commentaire de D. VAN MAL-MAEDER, La fiction, 2007, p. 58-59 (pour une récente traduction de cette déclamation: J. PINGOUD, A. ROLLE ET D.VAN MAL-MAEDER, Déclamations et intertextualité. Discours d'école en dialogue, Echo, Peter Lang, Berne, 2020); voir également, les exemples cités au chapitre III de ce même ouvrage, dont le caractère souvent poussif ou excessif a peut-être pu prêter à sourire.

15. «Our House is on Fire », Fridays For Future, mise en ligne le 22 avril 2020, consulté en septembre 2021 et disponible à l'adresse https://www.youtube.com/watch? $\mathrm{v}=\mathrm{eT} 32 \mathrm{UFzA7E}$ \&\&ab_channel=FridaysForFuture.

16. "Because if everyone knew how serious the situation is and how little is actually being done, everyone would come and sit down beside us." ou encore "The grown-ups has failed us and since most of them including the press and the politicians keep ignoring the situation we must take action into our own hands starting today. " Présents dans son premier discours en suédois au concert de la grève du climat à Stockholm du 8 septembre 2018 et dans la prise de parole en anglais de Greta Thunberg lors de la marche pour le climat devant le Parlement suédois le 13 septembre 2018.

17. On peut par exemple se demander qui sont les "pompiers" dans l'analogie construite par Greta Thunberg lors de son discours au Parlement européen, alors que le message globalement porté est qu'il n'y a justement pas à espérer d'assistance extérieure.

18. Nous avons mentionné l'intertextualité entre "Our house is on fire » de Greta Thunberg et « Notre maison brûle et nous regardons ailleurs» de Jacques Chirac. C'est également le cas avec «I also have a dream » prononcé dans un discours en anglais par Greta Thunberg le 18 septembre 2019 au Congrès américain ou encore avec «Blah blah blah blah » dans un discours prononcé plus récemment par la militante le 28 septembre 2021 à Milan. 


\section{AUTEUR}

SÉBASTIEN CHONAVEY

Université libre de Bruxelles 